\title{
Rhétorique et dialectique dans la « Lettre de la prison de Birmingham » de Martin Luther King
}

Rhetoric and Dialectic in Martin Luther King's "Letter from Birmingham Jail"

\section{Michael Leff}

Traducteur : Sivan Cohen-Wiesenfeld

\section{(2) OpenEdition}

\section{Journals}

Édition électronique

URL : http://journals.openedition.org/aad/1067

DOI : 10.4000/aad.1067

ISSN : 1565-8961

Éditeur

Université de Tel-Aviv

Référence électronique

Michael Leff, «Rhétorique et dialectique dans la « Lettre de la prison de Birmingham » de Martin Luther King », Argumentation et Analyse du Discours [En ligne], 6 | 2011, mis en ligne le 15 avril 2011, consulté le 10 décembre 2020. URL : http://journals.openedition.org/aad/1067 ; DOI : https://doi.org/ 10.4000/aad.1067

Ce document a été généré automatiquement le 10 décembre 2020.

\section{c) (i)}

Argumentation \& analyse du discours est mis à disposition selon les termes de la licence Creative Commons Attribution - Pas d'Utilisation Commerciale - Pas de Modification 4.0 International. 


\title{
Rhétorique et dialectique dans la « Lettre de la prison de Birmingham » de Martin Luther King
}

\author{
Rhetoric and Dialectic in Martin Luther King's "Letter from Birmingham Jail"
}

\section{Michael Leff}

Traduction : Sivan Cohen-Wiesenfeld

\section{NOTE DE L'ÉDITEUR}

Cet article a paru en anglais dans Eemeren, Frans H. van, J. Anthony Blair, Charles A. Willard \& Francisca A. Snoeck Henkemans (eds). 2003. Proceedings of the Fifth Conference of the International Society for the Study of Argumentation (Amsterdam : Sic Sat), 671-677, et est publié en français avec l'autorisation de l'éditeur.

\section{Rhétorique, dialectique et appels à la crédibilité}

1 En passant d'une approche formelle à une perspective informelle ou dialectique, le domaine de l'argumentation a également adopté, souvent à son insu, certains des centres d'intérêts traditionnellement associés à la rhétorique. Tant que les arguments étaient envisagés selon un modèle déductif formel, les considérations sociales et contextuelles étaient considérées comme non pertinentes. Un argument devait être jugé sur son contenu et sur la validité des relations formelles entre ses différentes propositions, et le recours à des éléments contextuels tels que la crédibilité de l'argumentateur était perçu comme fallacieux. Cependant, avec les progrès de la logique informelle, l'essentialisme du modèle déductif formel a fait place à une conception plus pratique de l'argumentation, qui voit dans le débat une pratique 
sociale, et englobe des considérations sur les personnes qui y participent et les circonstances qui l'entourent. Le recours au contexte, jadis catégoriquement rejeté au titre d'argument fallacieux, a été repensé comme une stratégie ou un schème dont l'emploi peut être légitime, et la logique informelle (ou dialectique, selon la dénomination que certains ont attribué à cette approche nouvelle) s'est alors tournée vers des questions qui relèvent clairement du domaine traditionnel de la rhétorique : en effet, des éléments tels que le moment, le lieu, la situation, les participants etc. ont toujours été considérés comme pertinents, sinon comme nécessaires, dans les études de rhétorique.

2 Pour illustrer cet intérêt pour des sujets qui relèvent de la rhétorique (et ses limites), je voudrais me référer à un récent article de Trudy Govier (1999), qui aborde le problème de la crédibilité dans la perspective des développements actuels de la recherche en logique informelle. L'auteur y traite de la version tu quoque de l'argument ad hominem et tente de démontrer que, contrairement à la conception qu'en propose le «traitement logique standard», celle-ci n'est pas toujours fallacieuse. Elle part de l'idée qu'un argument est plus qu'un simple assemblage de prémisses et de conclusions dans la mesure où il est toujours également une activité sociale impliquant un argumentateur et un public. Il en résulte que la relation entre l'orateur et son auditoire intervient dans l'évaluation de la qualité de l'argument. Or, pour que le public prenne l'argument de l'orateur au sérieux, il doit considérer celui-ci comme crédible. Govier affirme que deux dimensions au moins entrent dans l'évaluation de la crédibilité : une dimension épistémique (l'argumentateur possède-t-il une connaissance suffisante de la question traitée ?), et une dimension éthique (l'argumentateur n'est- il pas hypocrite et «fait-il sincèrement ce qu'il ou elle semble faire " ?). L'allégation tu quoque soulève, d'après Govier, une question pertinente en ce qui concerne la dimension éthique, car si une personne se contredit dans ses discours ou s'exprime dans un sens et agit dans un autre, l'auditoire a des raisons de penser qu'il ou elle ne croit pas vraiment aux propositions avancées par l'argument, et peut, par conséquent, se demander si l'argumentateur est sincère, voire même sincèrement engagé dans le processus d'argumentation. Les allégations tu quoque ne sont donc pas systématiquement fallacieuses parce que, bien que "sans rapport avec le contenu propositionnel de l'argument d'origine », elles portent « sur ses présuppositions sociales » et «mettent en cause la force qu'exerce l'argument sur l'auditoire. Ce point met évidemment l'accent sur le fait que la force d'un argument pour un auditoire donné dépasse largement son contenu propositionnel » (1999: 14-20).

Bien que le terme de rhétorique n'apparaisse à aucun point de cet article, je pense que la plupart des rhétoriciens le trouveraient intéressant et considéreraient qu'il touche à leurs propres préoccupations. En effet, les questions de base qu'il aborde se réfèrent à des thèmes courants en rhétorique tels que la crédibilité de l'argumentateur, le rôle de l'auditoire, la relation sociale entre l'orateur et son public, et la force de l'argument par rapport à un auditoire donné. L'étude de Govier révèle donc une affinité entre la logique informelle telle qu'elle est conçue actuellement et la rhétorique traditionnelle, même si elle ne reconnaît pas explicitement ce lien. Cependant, une fois notée cette affinité, on peut également relever des points de divergences entre les deux approches. Cet exercice devrait pouvoir servir de guide à un travail d'échange et de transfert fécond entre les domaines de la logique informelle et de la rhétorique. 
4 En premier lieu, l'intérêt que Govier affiche à l'égard de la crédibilité est à la fois plus ciblé et plus restreint que celui des rhétoriciens. Elle limite en effet son attention au rôle joué par celle-ci dans l'inférence logique et met l'accent sur le versant négatif du problème; elle ne considère pas la crédibilité comme une ressource argumentative mais comme une limitation à la force d'un argument; sa préoccupation est de déterminer dans quel cas un auditoire doit raisonnablement disqualifier un argument en raison de l'inconsistance de l'argumentateur. Le rhétoricien part d'un point de vue différent, qui pose la crédibilité comme un élément constructif de l'argumentation, un mode d'argumentation (par l'ethos) qui œuvre en coordination avec la preuve logique. Dans cette perspective rhétorique, la crédibilité présente des aspects plus nombreux et plus complexes que les deux dimensions retenues par Govier. Comme l'a observé Alan Brinton, la conception de l'ethos comprend au moins les éléments suivants: « compétence sur le sujet traité, bonnes intentions, partage des valeurs, des intérêts et des présuppositions de l'auditoire, fidélité au vrai, probité». Cette liste inclut les critères de Govier mais les dépasse largement en ce qui concerne l'importance accordée aux aspects positifs du caractère de l'orateur. Ce que veut le rhétoricien, c'est un argumentateur qui, comme le dit Brinton, incarne «l'ethos (le caractère) général de la société », quelqu'un sur qui "nous pouvons compter pour exprimer nos valeurs partagées, penser dans les termes de nos présomptions communes, exercer un bon jugement et parler en notre nom» (1985: 55). L'ethos rhétorique résulte donc de l'incarnation des valeurs culturelles, et ce but indique un intérêt pour la personne de l'orateur qui n'existe ni dans la logique ni dans la dialectique.

Deuxièmement, en accord avec l'orientation de la logique informelle, Govier étudie la crédibilité en rapport avec la croyance justifiée, alors que la rhétorique délibérative, genre dans lequel le caractère de l'orateur joue le rôle proéminent, prend fréquemment pour but l'action plutôt que la croyance (Brinton 1985: 248-251). Ce déplacement téléologique complique la tâche argumentative, puisqu'il y ajoute une dimension sociale et volontariste importante. Les rhéteurs engagés dans un discours délibératif doivent souvent négocier l'ambiguïté et la tension qui se crée entre les principes acceptés par l'auditoire et la façon dont il perçoit un cas particulier. Cette tension est suggérée par les topiques propres à la rhétorique délibérative, l'utile et l'honorable, et le rhéteur doit être capable, en réponse, de «jauger » l'auditoire et de démontrer sa capacité (forme de phronesis ou prudentia) à établir un équilibre entre des situations particulières et des principes plus durables. Cette aptitude ne répond pas à des normes fixes et abstraites, mais se manifeste en se déployant concrètement à travers la performance délibérative. Ainsi, dans la mesure où le rhéteur réussit cette performance, il ou elle réalise (enacts) le type de caractère approprié au jugement délibératif ; la réalisation ${ }^{1}$ (enactment) apparaît dès lors comme un aspect important de l'ethos rhétorique.

6 Un des problèmes typique de la rhétorique délibérative se pose lorsque la croyance et l'acte volontaire ne sont pas en harmonie, quand un auditoire adhère à certains principes mais ne parvient pas à les mettre en pratique. Ici, l'ad hominem dialectique se trouve inversé, et c'est l'auditoire et non l'orateur qui doit rendre compte de son manque de cohérence. Normalement, une argumentation de cet ordre est délicate et difficile parce que les auditoires ne reconnaissent pas facilement leur illogisme, et un argumentateur qui doit rendre apparente cette discordance et la mettre en évidence aux yeux de l'auditoire doit effectuer un recadrage général de la situation. C'est-à-dire 
qu'il doit évoquer une nouvelle perspective qui met en lumière les incohérences refoulées ou non détectées, et ouvre la voie à de nouvelles possibilités argumentatives. L'évocation est donc un autre aspect distinctif de la rhétorique.

7 En utilisant l'article de Govier comme point de référence, j'ai donc repéré trois traits caractéristiques, l'incarnation, la réalisation (enactment) et l'évocation, qui distinguent l'approche rhétorique de l'argumentation de celle de la logique informelle et de la dialectique contemporaines. Je voudrais maintenant expliciter ces dimensions de l'argumentation pour rendre la sensibilité rhétorique et son dispositif plus accessibles à ceux qui se spécialisent dans l'étude de l'argumentation. Dans ce but, plutôt que me livrer à un exposé théorique, je présenterai une étude de cas détaillée. Cette stratégie est conforme à la perspective rhétorique, et pour en expliquer les raisons, je me référerai une dernière fois à l'essai de Govier en y relevant une autre différence de tendance entre les rhétoriciens et les spécialistes de logique informelle.

La prise en compte de l'argument to quoque étaye une perspective générale abstraite: Govier s'intéresse, bien sûr, à la compréhension du contexte social et est sensible aux cas particuliers, qu'elle utilise comme une source de preuve et comme une mise à l'épreuve de son analyse. Néanmoins, elle considère systématiquement le tu quoque comme un type abstrait d'inférence argumentative, et se préoccupe beaucoup moins du contexte des arguments particuliers que des caractéristiques contextuelles qui participent généralement de la production de l'argument. En rhétorique, l'analyse reste liée de beaucoup plus près aux actes spécifiques d'argumentation et aux contextes dans lesquels ils apparaissent. Les arguments rhétoriques étant enracinés dans un cas particulier et dirigés vers lui, la force d'un argument peut difficilement être comprise ou évaluée en dehors de toute référence à ce cas particulier. Comme l'a noté Brinton : «Une des caractéristiques de la rhétorique, en contraste avec la logique, est qu'elle exige de s'attacher au particulier » $(1985: 56)$.

9 Les dialecticiens qui s'approprient à présent consciemment les techniques et les perspectives de la tradition rhétorique deviennent de plus en plus sensibles à ce point. Comme le révèlent les travaux de Walton, Tindale et d'autres, ils se contentent moins de simples exemples de manuels et sont plus enclins à entreprendre des analyses détaillées de cas réels. L'exemple le plus frappant de cette évolution est celui de van Eemeren et Houtlosser, qui proposent une lecture exhaustive et élaborée d'un texte hollandais classique, «L'apologie de William le Silencieux », pour étayer leur recherche sur l'argument rhétorique. Dans la suite de cet article, je voudrais offrir une contrepartie à cette étude en analysant un texte américain classique, la «Lettre de la prison de Birmingham » de Martin Luther King.

\section{La Lettre de la prison de Birmingham : contexte}

Au début du mois de janvier 1963, la Conférence des Etats du Sud (SCLC), l'organisation pour les droits civiques dirigée par le révérend Martin Luther King, prit pour cible la ville de Birmingham, dans l'Alabama, pour y mener une campagne d'action directe nonviolente. De telles campagnes se déroulaient depuis plusieurs années dans le sud des Etats-Unis, et elles comprenaient des rallyes, des marches, des boycotts, des sit-in et autres tactiques similaires ayant pour but de protester et, en définitive, d'éliminer la ségrégation raciale et autres formes de discrimination. Birmingham était une cible particulièrement importante. Ce n'était pas seulement l'une des plus grandes villes du 
sud, mais également un bastion de l'opposition à l'intégration raciale. La ville possédait un long passé de répression souvent brutale de ses citoyens noirs, et le Ku Klux Klan et d'autres organisations en faveur de la suprématie blanche y avaient si souvent usé de violence qu'elle était parfois surnommée «Bombingham». Le Gouverneur de l'Alabama, George Wallace, avait gagné les élections sur un programme de "ségrégation éternelle ", et le Commissaire de la Sûreté publique de la ville était Eugene «Bull» O'Connor, qui personnifiait la résistance dure et obstinée au mouvement des droits civiques. Le mouvement lui-même n'y avait pas enregistré de victoire majeure, et la campagne de Birmingham représentait donc un test critique quant à sa capacité à reprendre de l'élan pour réussir à surmonter l'une de ses sources d'opposition les plus puissantes.

11 Les choses se compliquaient encore en raison de la situation politique interne de Birmingham. Anxieux de restaurer l'image mise à mal de la cité et d'éliminer Bull Connor, un groupe de Blancs modérés avaient réussi à faire réformer le système de gouvernement de la ville, et une élection municipale devait avoir lieu en mars. Connor faisait partie des candidats, et la SCLC, craignant qu'un effort de protestation puisse créer une réaction négative en faveur de Connor, avait décidé de suspendre l'action jusqu'après l'élection. Les résultats de celle-ci s'avérèrent cependant indécis. Bull Connor et le candidat plus modéré, Albert Boutwell, émergèrent tous deux en tête, mais aucun d'entre eux n'obtint la majorité, et on dut programmer un second tour pour le 2 avril. Une fois de plus, la SCLC attendit les élections. Boutwell gagna, et le 3 avril, la SCLC lança sa campagne d'action directe non-violente.

12 Celle-ci ne démarra pas sous de bons augures. Contrairement aux attentes de King, seule une poignée de protestataires se joignit aux manifestations, et peu étaient prêts à faire de la prison. La SCLC avait prévu d'engendrer une crise en emplissant les prisons au-delà de leur capacité, mais au bout de huit jours, à peine plus de 150 personnes avaient été arrêtées, et de nouveaux volontaires devenaient de plus en plus difficiles à trouver (Branch 1998: 727-728). La couverture de l'événement par la presse ne répondait pas non plus aux attentes qu'on avait mises en elle, et les réactions à la campagne y étaient généralement défavorables. Le Washington Post considérait que le programme d'action directe aurait dû être suspendu afin de laisser à l'administration Boutwell une période d'installation raisonnable, et il estimait douteuse l'utilité des manifestations. Le procureur général Robert Kennedy pensait que l'effort déployé était inopportun, et même le journal noir local le rejetait comme "nocif et dépourvu de valeur » (Branch 1988 : 737, Bass $2001:$ 104-105). Comme l'a observé David Garrow, il y avait

au sein d'une partie importante de l'électorat, comme les pasteurs noirs, certains professionnels, les Blancs locaux les plus sympathisants et l'administration Kennedy, le sentiment que la victoire de Boutwell était une raison suffisante pour repousser à plus tard les manifestations. Ces groupes partageaient l'espoir qu'une fois l'administration modérée en place, aussi bien les négociants que l'administration municipale accéderaient à certaines des demandes du mouvement sans qu'il y ait besoin de manifester $(1986: 238)$.

13 Un problème supplémentaire surgit lorsque les procureurs de la ville réussirent à obtenir une injonction de la cour fédérale interdisant à King et à ses partisans de sponsoriser ou d'encourager des manifestations, ou d'y participer sans avoir préalablement obtenu une autorisation municipale. Les dirigeants de la SCLC étaient généralement peu disposés à violer les ordres de la cour fédérale, qu'ils considéraient 
comme un allié crucial. Dans ce cas, cependant, accepter l'injonction revenait pratiquement à stopper prématurément la campagne d'action directe, et King résolut de la violer lui-même et de se mettre en état d'arrestation dans l'espoir que cet « acte de foi " relancerait le mouvement (McWorther 2001: 355). Pour des raisons symboliques, il attendit le Vendredi Saint (12 avril 1963), et ce jour-là, dirigea une marche à travers les rues de la ville et fut arrêté. Refusant de se faire libérer sous caution jusqu'au 19, King resta en prison pendant huit jours (Branch 1988 : 734-747, Garrow 1986 : 241-246).

Le matin qui suivit son arrestation, le Birmingham News publia une courte lettre ouverte signée par huit éminents membres du clergé. Considérés comme modérés sur les problèmes raciaux, ceux-ci venaient juste de signer trois mois plus tôt une autre lettre ouverte appelant directement les citoyens de l'Alabama à obéir à l'injonction de la Cour suprême d'abolir les distinctions raciales dans les écoles, et critiquait indirectement la politique de mépris du Gouverneur Wallace. Dans cette seconde lettre, ces hommes d'église prônaient également la modération et l'obéissance à la loi, mais à présent, leur critique était dirigée implicitement contre King et son programme d'action directe non-violente. La lettre affirmait que la ville s'orientait vers une nouvelle approche des problèmes raciaux, constructive et réaliste, et que les manifestations " conduites en partie par des outsiders » étaient à la fois peu sages et peu opportunes. Les problèmes raciaux devaient être résolus à l'aide de "négociations ouvertes et honnêtes », et rien n'avait été accompli par des moyens d'action qui incitaient «à la haine et à la violence, aussi pacifiques qu'elles aient pu être dans le sens technique du terme ». Les auteurs félicitaient l'ensemble de la communauté, et en particulier les fonctionnaires chargés de l'application de la loi, pour avoir géré la situation dans le calme, et concluaient par un appel aux habitants noirs de Birmingham à retirer leur soutien aux manifestations et à résoudre leurs griefs au tribunal et à travers un processus de négociation (Bass $2001: 235-236$ ).

15 L'avocat de King lui fit parvenir le journal en fraude dans la cellule de sa prison, et selon la version établie des faits, King commença immédiatement à rédiger sa réponse (écrite d'abord dans les marges du journal puisqu'il n'avait pas d'autre papier). La version publiée de la lettre date du 16 avril, et, bien que nous ayons de bonnes raisons de penser que le document n'a pas été terminé avant la libération de King, le ton et la texture du texte confortent l'impression qu'il a été composé de l'intérieur d'une cellule de prison (Bass $2001:$ 131-152, Branch 1988: 737-745). La lettre avait eu peu d'impact dans l'immédiat mais, avant la fin de l'année 1963, elle avait largement circulé, à la fois sous la forme pamphlet et de publication dans des magazines. Elle gagna vite une audience large et enthousiaste et finit par prendre place parmi les textes canoniques de la rhétorique politique et dans les anthologies de littérature américaines².

\section{La Lettre : aspects dialectiques}

En tant qu'étudiant à l'université de Boston, King avait été fasciné par la philosophie d'Hegel, non en raison de sa métaphysique ou de son éthique, qu'il rejetait, mais à cause de sa méthode dialectique. Le modèle hégélien de paires d'oppositions et de résolution synthétique semblait convenir à son propre tempérament et à ses tendances intellectuelles, et l'un de ses professeurs, L. Harold Dewolf, a noté que « quelque soit le sujet traité, King ne s'est jamais lassé de passer d'une thèse à une antithèse, et de là à 
une synthèse » (Garrow 1986 : 46). Cette sensibilité dialectique apparaît clairement dans la "Lettre de la prison de Birmingham ", et en fait, le texte peut être caractérisé comme « dialectique » dans plusieurs sens du terme.

Tout d'abord et de la façon la plus évidente, le texte fonctionne à travers une série d'arguments opposés. Mis à part une brève introduction, une conclusion et deux sections qu'il désigne sous le terme de " confessions ", la lettre consiste en une série de réponses aux arguments attribués aux huit membres du clergé. La structure suivante révèle clairement ce modèle :
A. Introduction
B. Réfutation
1. de l'argument selon lequel King est un outsider
2. du fait que lui et ses partisans devraient négocier plutôt que manifester
3. que les manifestations sont inopportunes (première confession : sa déception
face aux Blancs modérés)
4. que l'action directe non-violente précipite la violence
5. que les problèmes raciaux se résoudront d'eux-mêmes avec le temps
6. que King et ses partisans soient extrémistes (deuxième confession : la déception
de King vis-à-vis du clergé blanc)
7. que la police de Birmingham mérite des éloges
c. Conclusion

18 Au fil d'une une analyse serrée (close reading) du texte, la structure s'avère beaucoup plus subtile que cette réduction schématique ne l'indique, mais cet aperçu n'en représente pas moins avec précision l'importance des paires dialectiques d'arguments et de contre-arguments.

Le texte est également dialectique dans le sens où l'argumentation s'y développe sous une forme dialogique. Alors que la lettre ouverte des huit ecclésiastiques n'était pas adressée à une ou des personnes spécifiques, celle de King débute par leurs noms suivis de la salutation "Mes chers confrères ecclésiastiques». Et le premier paragraphe continue sur le même modèle d'adresse directe, le «je » de King répondant au « vous » représentant les auteurs de la lettre précédente. Cette relation s'exprime clairement à la fin du paragraphe : «mais puisque je pense que vous êtes véritablement des hommes de bonne volonté et que vos critiques sont sincères, je voudrais répondre à vos affirmations dans des termes, je l'espère, sereins et raisonnables" (84) ${ }^{3}$. Ce type d'adresse se poursuit tout au long de la lettre, et il est spécialement flagrant dans les phrases qui marquent une nouvelle section du texte. Presque toutes ces phrases attribuent une position spécifique aux ecclésiastiques, auxquels King s'adresse à la seconde personne : "Vous exprimez une grande inquiétude à l'idée que nous sommes disposés à enfreindre la loi » (89). Ce caractère dialogal est parfois renforcé par l'utilisation de questions rhétoriques: "Vous pouvez effectivement demander "Pourquoi une action directe? Pourquoi ces sit-in, ces marches etc.? La négociation n'est-elle pas un meilleur moyen d'action ?" Vous avez absolument raison d'appeler à la négociation $»(86)^{4}$. La lettre prend alors une forte orientation dialogale.

La dialectique se caractérise également par la fait que les interlocuteurs sont supposés répondre à une exigence de rationalité (dans le sens d'obtempérer au "raisonnable »), et King évoque ce standard aussi bien implicitement qu'explicitement. Dans le passage du paragraphe d'ouverture que je viens de citer, il s'engage à répondre sur un mode patient et raisonnable, et formule donc tous ses arguments sous une forme claire et 
logique. De plus, comme je l'expliquerai par la suite, le texte renforce implicitement cette attitude par sa retenue scrupuleuse et son ton raisonnable.

Somme toute, la Lettre présente son auteur comme un avocat discipliné, engagé dans un débat rationnel avec un adversaire spécifique, sur des positions bien définies et nettement opposées. A cet égard, la Lettre présente un caractère dialectique, et elle invite à une analyse argumentative fine de la part des dialecticiens et des spécialistes de logique informelle contemporains, analyse qui promet d'être fructueuse. Cependant, elle constitue également un appel à l'action, et illustre parfaitement les trois dimensions spécifiques de l'argumentation rhétorique: l'incarnation, la réalisation (enactment) et l'évocation. C'est cette voie que j'emprunterai à présent, pour étudier le texte dans une perspective rhétorique.

\section{L'incarnation rhétorique}

Bien que la Lettre de King s'adresse littéralement aux huit ecclésiastiques de Birmingham, elle n'a jamais été expédiée personnellement à aucun d'entre eux et, de fait, ils ne constituaient pas son auditoire-cible. Ils fonctionnaient comme une synecdoque, comme une représentation du public plus large que King souhaitait atteindre, et sa décision de répondre à leur lettre ainsi que la manière de le faire étaient toutes deux d'ordre stratégique. Le succès de la campagne de Birmingham, et des efforts de la SCLC en général, dépendait fortement du soutien des Blancs modérés, ceux qui avaient d'ores et déjà tendance à désapprouver la ségrégation raciale et à se sentir mal à l'aise face à l'écart creusé entre leurs valeurs fondamentales et la politique publique discriminatoire qui régnait alors dans le Sud. La lettre des huit ecclésiastiques offrit à King l'opportunité d'incarner cet auditoire-cible et de se référer directement à leurs préoccupations, sans que l'occasion ni les sujets abordés ne paraissent avoir été fabriqués intentionnellement. De plus, comme l'a noté Richard Fulkerson (1979 : 124), le choix d'un groupe spécifique comme public ostensible de la lettre ouverte a permis à King de développer un ton personnel et de projeter sa propre personnalité d'une façon qui n'aurait pas été possible dans un document dépourvu de destinataire particulier.

King n'avait pas besoin de construire une relation synecdoctique entre lui et le mouvement pour les droits civiques. Ce lien existait déjà dans l'esprit de l'auditoire, et le problème de King sur le plan rhétorique n'était donc pas d'incarner le mouvement dans sa persona, mais bien de construire une persona qui incarne les valeurs et les intérêts de son public-cible. La plus grande partie du texte se consacre à cette tâche, et les efforts de King se développent dans plusieurs directions. Au niveau des assertions directes, il s'associe aux principes américains fondamentaux d'égalité et de liberté, endosse « le rêve américain » et fait l'éloge de « ces grands réservoirs de la démocratie creusés en profondeur par les Pères Fondateurs lorsqu'ils formulèrent la Constitution et la Déclaration d'Indépendance» $(100)^{5}$. De même, et de manière encore plus explicite, King s'inscrit dans la foi chrétienne :

Avec une profonde déception, j'ai pleuré le laxisme de l'église. Mais soyez sûrs que mes larmes étaient des larmes d'amour... Oui, j'aime l'Eglise ; j'aime ses murs sacrés. Comment pourrais-je faire autrement? Je me trouve moi-même dans une situation plutôt exceptionnelle : je suis fils, petit-fils et arrière petit-fils de prédicateurs (97) ${ }^{6}$.

Ici la présentation de King recouvre trois niveaux d'incarnation: le christianisme est concrétisé par la représentation de l'église comme espace matériel entouré de murs; 
King, venant d'une lignée reliée à cet espace, incarne son identité à l'intérieur de ces murs, et depuis cette position interne la déception que l'Eglise lui a causée ne peut se matérialiser qu'à travers des larmes d'amour. Tout ce travail de figuration présente King comme ayant les références appropriées pour critiquer l'Eglise de l'intérieur et la rappeler à ses propres idéaux.

King incarne également sa solidarité avec les valeurs américaines dominantes à travers l'utilisation d'arguments ad verecundiam. Le texte est parsemé de références à des figures d'autorité de l'histoire américaine, du savoir judéo-chrétien et de la tradition intellectuelle occidentale, comme l'apôtre Paul, Socrate, Reinhold Niebuhr, Thomas d'Aquin, Martin Buber, Paul Tillich, Jésus, le prophète Amos, Martin Luther, John Bunyan, Abraham Lincoln, Thomas Jefferson et T. S. Eliot. Il invoque ces références pour expliquer ses propres actions et les justifier. Pour prendre un exemple marquant, en réponse à l'accusation qui lui est faite d'être un outsider, il se reporte aux Ecritures pour trouver un précédent à son comportement :

De plus, si je suis à Birmingham, c'est parce que l'injustice s'y trouve. Tout comme les prophètes du huitième siècle quittèrent leur petit village pour porter la « Parole du Seigneur " par-delà les frontières de leurs terres natales; et tout comme l'apôtre Paul quitta son petit village de Tarse pour porter l'évangile de Jésus Christ dans presque tous les hameaux et villes du monde gréco-romain, moi aussi, mon devoir est de porter l'évangile de la liberté par-delà ma terre d'origine (84-85) ${ }^{7}$.

King s'efforce évidemment d'enrayer la perception qu'on a de lui à Birmingham, qui en fait un outsider au sens littéral mais aussi idéologique du terme, un homme dont les attitudes de base s'écartent des conceptions américaines considérées comme respectables. L'argument ad verecundiam lui rend un double service pour contrer cette image. Tout d'abord, en citant des figures qui font foi dans le domaine de la croyance et de la religion, King s'associe à des autorités inconditionnellement reconnues par son auditoire-cible, suggérant ainsi son affiliation à cet auditoire. Deuxièmement, les paroles et actes de ces figures respectées, dans la mesure où elles semblent identiques ou similaires à celles de King, prennent valeur d'exemples qui justifient sa position et lui accordent une place dans l'espace de l'orthodoxie judéo-chrétienne. Si Amos, Paul, Socrate et même Jésus, se conduisent en agitateurs, il en résulte que l'agitation visant à dévoiler et surmonter l'injustice ne menace pas la tradition commune, mais permet au contraire de la renouveler et de la maintenir dans son intégrité.

\section{La réalisation rhétorique}

L'incarnation et la réalisation (enactement) sont des phénomènes rhétoriques qui entretiennent entre eux une liaison étroite. Dans la plupart des textes, et en particulier dans ceux qui sont bien construits, ils se chevauchent, si bien qu'un travail attentif d'interprétation s'avère nécessaire pour les distinguer. Cependant, comme j'espère le montrer maintenant, cette distinction est importante à établir. L'incarnation résulte de ce que dit le texte, de ses assertions et de ses arguments. La réalisation résulte de ce que fait le texte. Pour comprendre cette distinction, nous devons concevoir le texte argumentatif non comme un produit inerte, mais comme un champ d'action qui, en se développant, construit des représentations et des relations - une sorte de microcosme du monde social auquel il s'adresse. Dans ce sens, les textes construisent une image de l'auteur, une image de l'auditoire, et une relation (ou un ensemble de relations) entre les deux. Van Eemeren et Houtlosser, bien qu'ils n'utilisent pas ma terminologie, 
proposent un excellent exemple d'une telle relation dans leur analyse d'un publireportage de la Société Shell lorsqu'ils notent que le texte s'adresse à son public comme «un père parlerait à son enfant" (1999: 490). Bien sûr, le texte n'énonce jamais explicitement cette relation ; Van Eemeren et Houtlosser l'infèrent à partir du ton et de l'attitude qui s'affichent dans le déroulement de l'argumentation, et une fois qu'ils ont mis à jour la relation parent/enfant figurée dans le texte, ils peuvent porter certains jugements importants sur le caractère et les motifs de son auteur. Le texte se comporte d'une certaine manière envers l'auditoire, et à partir de ce comportement, celui-ci peut faire des inférences sur son auteur.

Dans la Lettre de King, le processus de réalisation (enactment) est complexe et subtil, et il aboutit à une représentation complexe mais cohérente du caractère de l'auteur. Celuici est dépeint comme énergique, actif, se considérant comme engagé par ses principes et agissant en accord avec eux, mais aussi comme une personne posée, équilibrée, raisonnable et retenue. L'image qui prédomine est celle d'une énergie contrôlée, et cette image est conçue pour dissiper l'accusation lancée contre King selon laquelle il serait un radical qui manque de jugement et agit sans se préoccuper des conséquences de ses actes.

29 A travers la série des réfutations, le texte met en œuvre (enacts) un entendement équilibré au moyen de ce que Fulkerson (1979: 127) appelle un «modèle duel ». King répond aux allégations portées contre lui, d'abord à un niveau pratique immédiat puis sur le plan des principes, et à mesure que le modèle se développe, le lecteur est témoin de la manière dont King fait preuve du type de discernement le plus approprié à la délibération, celui qui tient compte à la fois des principes et des cas particuliers, des questions d'utilité et de dignité. La première des réfutations de King offre une illustration claire de ce point. En réponse à l'accusation selon laquelle il serait un outsider, King commence par expliquer que la branche affiliée de la SCLC à Birmingham avait réclamé son assistance, et qu'il était donc « là, avec plusieurs membres de mon équipe, parce que nous avons été invités à venir ». Mais la question ne s'arrête pas là car, au-delà de ces considérations particulières, se pose également un impératif moral qui conduit King à affronter l'injustice, comme le firent les prophètes hébreux et l'apôtre Paul. Et, pour replacer le problème dans une perspective encore plus large, King reconnaît

l'interrelation entre tous les pays et Etats... L'injustice, où qu'elle soit, est une menace pour la justice partout ailleurs. Nous sommes pris dans un réseau de relations mutuelles auquel nous ne pouvons pas échapper, liés par une destinée unique. Tout ce qui affecte directement l'un affecte directement tous les autres $(85)^{8}$.

30 Par conséquent, aussi bien au niveau du cas particulier que sur le plan des principes éthiques généraux, King ne doit pas être considéré comme un outsider; sa présence à Birmingham est à la fois appropriée et légitime.

Les deuxième, troisième et quatrième séquences de réfutation utilisent également cette double structure, mais c'est dans la sixième, dans laquelle King répond à l'accusation d'extrémisme, que cette technique trouve son expression la plus puissante. Dans la quatrième séquence, King commence par exprimer sa surprise que l'on puisse le qualifier d'extrémiste, alors qu'il se trouve en réalité «à mi-chemin entre deux forces opposées de la communauté noire ». D'un côté, il y a ceux qui se contentent d'accepter l'injustice sans rien faire, et de l'autre, les nationalistes qui réagissent à l'injustice par 
la haine et l'amertume et sont " dangereusement proches d'un plaidoyer en faveur de la violence ». Entre ces deux extrêmes, la complaisance et la colère du désespoir, King offre «l'excellente voie » de la protestation non-violente, et il affirme sa déception de voir que cette position puisse être rejetée comme extrémiste. Cependant, en poursuivant sa réflexion à ce sujet, il trouve peu à peu « quelque satisfaction à être considéré comme extrémiste. Jésus n'était-il pas extrémiste dans l'amour - "Aimer vos ennemis, bénissez ceux qui vous maudissent, priez pour ceux qui vous méprisent" ». Cet argument ad verecundiam est développé à travers une longue liste de figures héroïques (comprenant Amos, Paul, Martin Luther, John Bunyan, Abraham Lincoln et Thomas Jefferson) reliées à des citations célèbres exprimant des idées extrêmes. Et King conclut que la question n'est pas de savoir si «nous voulons être des extrémistes ", mais bien plutôt si nous serons extrémistes pour l'amour et la justice ou bien pour la haine et l'injustice (92-94).

Comme l'ont notés d'autres commentateurs, (par exemple Fulkerson 1979:128), ce passage fait la distinction entre l'extrémisme compris comme un positionnement au sein d'un spectre de positions existantes, et l'extrémisme compris en termes d'intensité de conviction. Dans le premier cas, King n'est pas un extrémiste, mais plutôt un modéré au tempérament dialectique, puisque son point de vue réalise un équilibre entre les forces antithétiques de l'apathie et de la violence, et en offre une synthèse constructive. Dans le second, cependant, King est un extrémiste puisqu'il s'engage avec passion pour le principe de l'action contre l'injustice et de son éradication, mais comme le démontre les témoins historiques cités par King, cette forme d'extrémisme n'est pas nécessairement mauvaise puisqu'elle peut contribuer à préserver l'héritage culturel. Le mouvement global de ce passage reflète une combinaison de retenue et d'engagement qui irradie favorablement le personnage de l'auteur ainsi que le caractère du mouvement auquel il est identifié.

Une autre caractéristique notable de ce passage réside dans le fait que King, lorsqu'il est confronté à l'accusation d'extrémisme, réagit non pas avec colère ou indignation, mais en exprimant sa déception. Cette sorte de retenue verbale est récurrente tout au long de la Lettre, et le choix des termes à cet égard vient ajouter à la consistance de l'image élaborée par d'autres aspects du texte. Mais l'énergie contenue de King est figurée encore plus puissamment par la structure de certaines de ces phrases, dont la syntaxe réalise (enacts) la retenue.

Dans la troisième séquence de réfutation de la lettre, King offre une réponse soigneusement modulée à l'accusation selon laquelle les manifestations sont inopportunes. Les Afro-américains, rappelle-t-il à ses lecteurs, attendent l'obtention de leurs droits depuis déjà 340 ans, et il n'est pas étonnant qu'ils commencent à s'impatienter. "Peut-être ", ajoute-t-il "est-il facile pour ceux qui n'ont jamais eu à souffrir des blessures occasionnées par les flèches de la ségrégation de dire "Attendez" " ${ }^{9}$ :

Mais quand vous avez vu des populaces vicieuses lyncher à volonté vos pères et mères, noyer à plaisir vos frères et sœurs ; quand vous avez vu des policiers pleins de haine maudire, frapper, brutaliser et même tuer vos frères et sœurs noirs en toute impunité; quand vous voyez la grande majorité de vos vingt millions de frères noirs étouffer dans la prison fétide de la pauvreté, au sein d'une société opulente; quand vous sentez votre langue se nouer et votre voix vous manquer pour tenter d'expliquer à votre petite fille de six ans pourquoi elle ne peut aller au parc d'attractions qui vient de faire l'objet d'une publicité à la télévision; quand vous voyez les larmes affluer dans ses petits yeux parce qu'un tel parc est fermé aux 
enfants de couleur; quand vous voyez les nuages déprimants d'un sentiment d'infériorité se former dans son petit ciel mental ; quand vous la voyez commencer à oblitérer sa petite personnalité en sécrétant inconsciemment une amertume à l'égard des Blancs; quand vous devez inventer une explication pour votre petit garçon de cinq ans qui vous demande dans son langage pathétique et torturant: «Papa, pourquoi les Blancs sont si méchants avec ceux de couleur?»; quand, au cours de vos voyages, vous devez dormir nuit après nuit sur le siège inconfortable de votre voiture parce que aucun motel ne vous acceptera ; quand vous êtes humilié jour après jour par des pancartes narquoises : «Blancs ", " Noirs "; quand votre prénom est «négro» et votre nom «mon garçon» (quel que soit votre âge) ou "John"; quand votre mère et votre femme ne sont jamais appelées respectueusement «madame »; quand vous êtes harcelé le jour et hanté la nuit par le fait que vous êtes un nègre, marchant toujours sur la pointe des pieds sans savoir ce qui va vous arriver l'instant d'après, accablé de peur à l'intérieur et de ressentiment à l'extérieur; quand vous combattez sans cesse le sentiment dévastateur de n'être personne; alors vous comprenez pourquoi nous trouvons si difficile d'attendre $(88-89)^{10}$.

Le trait le plus remarquable de cette phrase est sa longueur - 351 mots $^{11}$ d'après le compte de Fulkerson - qui en fait de loin la plus longue phrase du texte et probablement l'une des plus longues phrases de la prose anglophone contemporaine. Mais on doit également remarquer la syntaxe de cette phrase. En raison de sa structure centripète ou en forme de période, elle se complexifie sur le plan syntaxique par l'addition de propositions subordonnées qui précèdent la principale. Cette organisation suspend jusqu'à la fin l'accomplissement final de la phrase en tant qu'unité chargée de sens, si bien que, pour la comprendre, le lecteur doit attendre jusqu'aux douze derniers mots de clôture. De plus, dans la mesure où les subordonnées relatent une série de maux, d'insultes et d'outrages, l'ensemble de la phrase figure iconographiquement la difficile condition de l'Afro-américain (Klein 1981 : 30-47). Les lecteurs blancs n'ayant jamais souffert des «blessures occasionnés par les flèches de la ségrégation » doivent attendre pendant que cette longue liste de griefs continue à assaillir leur sensibilité, et ils font ainsi l'expérience, sous une forme indirecte, de la frustration des Afroaméricains. La phrase met en scène (enacts) et transmet cette expérience mieux que n'importe quel argument propositionnel n'aurait pu le faire.

Etant donné la longueur de la phrase, la tension qui monte à travers elle et l'intensité avec laquelle elle représente les effets du fanatisme, on aurait pu s'attendre à ce qu'elle se termine sur une note d'indignation et de colère, voire même sur une mise en accusation de ceux qui demandaient à King et à son peuple de patienter. Au lieu de cela, cependant, l'apogée vient sous la forme d'une litote adressée à l'auditoire blanc : « alors vous comprenez pourquoi nous trouvons si difficile d'attendre ». Dans cet exemple, la litote contribue sans doute à augmenter l'impact émotionnel de la phrase, mais c'est également une mise en scène frappante (enactment) de la retenue de King. En fait, il m'est difficile d'imaginer une représentation textuelle plus appropriée de la volonté de King de s'exprimer en termes raisonnables et modérés ${ }^{12}$.

Pour résumer, la réalisation (enactment) joue un rôle important dans l'argumentation de la Lettre de la prison de Birmingham. Pour atteindre son auditoire cible, King a besoin de dissiper la perception qu'on avait donnée de sa personne - celle d'un radical adonné à un mode d'action incontrôlé, et soutenant des points de vue qui vont à l'encontre du consensus établi dans la société américaine. C'est pourquoi le texte le présente systématiquement sous un jour différent, et il le fait non seulement par l'usage d'assertions explicites, mais également par la mise en scène (enactment) de sa 
capacité de jugement équilibré et tempéré, et en "parlant » d'une voix affligée et déterminée, mais en même temps retenue et raisonnable. A la fin de la Lettre, King énonce ce thème en deux phrases parfaitement équilibrée qui circonscrivent le personnage qu'il projette à travers le texte :

Si j'ai dit quoique ce soit dans cette lettre qui soit une exagération de la vérité et indique une impatience irraisonnée, je vous prie de me pardonner. Si j'ai dit quoique que ce soit dans cette lettre qui soit au-dessous de la vérité, et indique de ma part une patience susceptible de me faire prendre en patience quoi que ce soit en-deça de la fraternité, je prie Dieu de me pardonner $(100)^{13}$.

\section{L'évocation}

L'évocation opère à un niveau plus élevé de généralisation que l'incarnation ou la réalisation (enactment), puisqu'elle se réfère à la représentation et à l'appréhension d'une situation dans son ensemble. Dans leur récente étude sur la question, Walter Jost et Michael Hyde expliquent que l'évocation se produit à travers la réalisation d'un modèle par l'accumulation d'un ensemble de cas particuliers. Cette réalisation doit être pleine de vivacité, elle doit saisir la chose comme « un ensemble dans le cadre duquel tout prend sens ", et s'obtient à travers la persuasion (1997 : 23). Abordant ce sujet dans une perspective de dialecticien, Nicholas Rescher propose une analyse similaire de la force de persuasion rhétorique. La rhétorique, affirme Rescher, peut provoquer l'adhésion à travers une forme d'expression synthétique qui saisit et éclaire certains domaines de notre expérience pour attirer sur elles l'attention et les faire accéder à la pleine conscience. Ce processus implique le sens de l'intégration à un tout et résulte, dans une large mesure, de la force intrinsèque de ce qui est dit (Rescher: 1998). En d'autres termes, l'évocation recadre ou restructure la perception d'une situation parce qu'elle appelle à la reconnaître comme un ensemble, et comme quelque chose qui concorde avec notre héritage culturel, et cet appel est lié au pouvoir du langage utilisé dans l'effort de persuasion.

La Lettre de la prison de Birmingham exemplifie le mode de fonctionnement du processus évocateur. Elle s'adresse à un auditoire-cible de Blancs modérés qui, tout en ressentant qu'un fossé sépare leurs idéaux des pratiques discriminatoires de la société dans laquelle ils vivent, se méfient d'un changement radical, s'inquiètent des protestations qui violent les lois et accroissent les tensions, et s'inquiètent des agitateurs extérieurs qui pourraient utiliser des idéaux irréalistes pour bouleverser la stabilité de l'ordre social et politique existant. La rhétorique de King émousse ces craintes et permet d'établir une relation positive entre sa propre position et l'héritage de son auditoire. Comme l'a avancé E. Culpepper Clarck, King a su exploiter les attentes culturelles implicites de la situation et les transformer « en une métaphore dominante pour interpréter la désobéissance civile non-violente ». La Lettre transforme King d'un radical potentiellement immodéré et dangereux, en prophète rappelant son peuple au meilleur de lui-même et en dirigeant dont la voix « fait écho à la lutte judéo-chrétienne contre la servitude » (1993: 48 - 49).

Mais quelle est la relation entre l'évocation et l'argumentation ? Clarck suggère que ce lien n'est pas particulièrement puissant, puisque la force de la Lettre de la prison de Birmingham résulte du choix de la bonne métaphore au bon moment dans les bonnes circonstances. Ce point de vue, cependant, n'explique pas les modalités au gré desquelles King a pu déployer efficacement cette métaphore, question particulièrement 
importante si l'on considère l'image impliquée dans ce cas d'espèce. La voix prophétique vient de l'intérieur du peuple qu'elle critique; elle incarne ce qu'il y a de plus haut et de meilleur dans la société et appelle les autres à agir en fonction de normes partagées par le locuteur et ses auditeurs. Le prophète n'est pas un outsider ou un observateur, mais un membre de la tribu, et pour être un prophète parmi les Hébreux, il faut être un Hébreu. Et pour être un prophète parmi les Américains blancs modérés? Ce n'est pas un rôle dont King a hérité par la naissance, ni qu'il a acquis aisément. Il doit l'endosser par la persuasion, et sa lettre est bien conçue dans cet objectif. Elle construit des arguments qui lient l'auteur et l'auditoire même en présence d'un désaccord entre eux, et parle d'une manière qui met en scène (enacts) et incarne le personnage du bon délibérateur. Une fois qu'il a établi de façon plausible son rôle de délibérateur, King peut plus facilement se positionner comme quelqu'un qui parle de l'intérieur de la culture de son auditoire. Je ne veux pas dire par là que ce processus soit strictement linéaire, que l'argumentation soit le premier pas et que l'évocation ne puisse venir qu'une fois que les arguments ont accompli leur tâche. Les deux phénomènes semblent fonctionner ensemble sur un mode interactif et moins clairement délimité : à mesure que les arguments de King s'accumulent et se renforcent, le pouvoir évocateur du texte devient plus apparent; en même temps, à mesure que l'évocation gagne en puissance, les arguments de King acquièrent une clarté et une force plus grandes. Quel que soit l'ordre de cette relation, cependant, je pense qu'elle se développe clairement à l'intérieur du texte même, et que la remarquable capacité de King à parler efficacement en prophète à un auditoire blanc est d'une certaine façon liée au crédit qu'il parvient à obtenir en qualité de dialecticien.

Le processus que je viens de décrire est certes paradoxal, puisque la prophétie et l'argumentation sont ordinairement attribuées à des domaines d'activité différents. Mais peut-être le moment est-il venu pour les chercheurs en argumentation de mieux s'accommoder des paradoxes qui déplacent les catégories et stimulent des relations nouvelles et inattendues. Avec le déclin du modèle déductif formel et de l'essentialisme qui lui est associé, on peut difficilement s'attendre à ce que le dispositif critique reste coi et continue de soutenir nos vieilles hypothèses disciplinaires. C'est ainsi, par exemple, que l'étude de Trudy Govier sur l'argument tu quoque, centrée sur la logique, l'a conduite à découvrir « que la force d'un argument dépend à bon escient de quelque chose de plus que son contenu propositionnel» (1999: 20). De même, mon étude de la Lettre de la prison de Birmingham m'a conduit à conclure qu'il y a plus dans l'évocation rhétorique que le temps, le hasard et l'imagerie. Govier ne possède, à mon avis, pas encore une compréhension parfaite des éléments non-propositionnels qui contribuent à faire la force d'un argument, et, de mon côté, je confesse ma profonde ignorance de la manière dont l'argument dialectique influence la persuasion rhétorique et la rend possible. Mais une chose est certaine, c'est que nous avons quelque chose à apprendre l'un de l'autre, et je pressens que le processus sera plus rapide et plus efficace si nous nous attachons à des cas de figure - non à des cas simples ou clairement erronés, mais à ceux qui présentent les meilleurs pratiques d'argumentation. Ce sont ces cas d'espèce que nous devons essentiellement prendre en considération si nous voulons faire de la théorie de l'argumentation, non pas seulement un instrument visant à corriger les erreurs de raisonnement, mais aussi une ressource maniable et constructive qui permette de gérer les activités publiques des chercheurs et des citoyens. 


\section{BIBLIOGRAPHIE}

Bass S. Jonathan \& Martin L. King. 2001. Blessed are the peacemakers : Martin Luther King, Jr., eight white religious leaders, and the Letter from Birmingham Jail (Baton Rouge : Louisiana State University Press)

Brinton, Alan. 1985. « A rhetorical view of ad hominem », Australian Journal of Philosophy 63, 50-63 Brinton, Alan. 1986. « Ethotic argument ». History of Philosophy Quarterly 3, 255-258

Branch, Taylor. 1988. Parting the Waters : America in the King Years 1954-63 (New York : Simon and Schuster)

Culpepper, Clark E. 1993. The Schoolhouse Door : Segregation's Last Stand at the University of Alabama (New York : Oxford University Press)

Eemeren, Franz H. van \& Peter Houtlosser. 1999. « William the Silent's Argumentative Discourse », Eemeren, Franz H. \& al. (eds), Proceedings of the Fourth International Conference of the International Society for the Study of Argumentation (Sic Sat : Amsterdam), 168-171

Fulkerson, Richard. 1979. «The Public Letter as a rhetorical Form : Structure, Logic and Style in King's Letter from Birmingham Jail », Quarterly Journal of Speech, 65, 121-136

Garrow, David J. 1986. Bearing the cross : Martin Luther King, Jr., and the Southern Christian Leadership Conference (New York : W. Morrow)

Govier, Trudy. 1999. The Philosophy of Argument (Newport News, VA : Vale Press), Ch. 2. « Political speech, Oliver Sachs and the credibility concern », 13-24

Jost, W. \& M. J. Hyde (Eds.). 1997. Rhetoric and hermeneutics in our time (New Haven : Yale University Press)

King, Martin Luther. 2006. «Lettre de la geôle de Birmingham », La Recherche du Bonheur, accessible en ligne :www.larecherchedubonheur.com/article-4599652.htm [retiré le 19 novembre 2006]

King, Martin Luther. 1986. « Letter from Birmingham Jail », J. M. Washington (ed.). I Have a Dream : Writings and Speeches that Changed the World (San Francisco : Harper), 84-100

Klein, Mia. 1981. « The other beauty of Martin Luther King's Letter from Birmingham Jail », College Composition and Communication 32, 30-37

McWorther, Diane 2000. Carry me Home: Birmingham, Alabama. The Climatic Battle of the Civil Rights Revolution (New York : Schuster \& Schuster)

Rescher, Nicholas 1998. "The role of rhetoric in rational argumentation », Hansen, Hans V., Christopher W. Tindale \& A. Colman (eds). Argumentation and Rhetoric (St Catherines : OSSA) Tindale, Christopher W. 1999. Acts of Arguing : A Rhetorical Model of Argument (Albany : SUNY)

\section{NOTES}

1. Le terme d'enactment, qui désigne l'une des notions clés de cet article, n'a pas d'équivalent exact en français et nécessite, ainsi que le verbe to enact, des traductions différentes dans les 
occurrences où il est employé. Nous avons donc choisi, dans la plupart des cas, de signaler entre parenthèses le terme anglais (Note de la rédaction).

2. La version anglaise originale de la lettre de Martin Luther King de la prison de Birmingham est disponible en ligne sur: http://www.africa.upenn.edu/Articles_Gen/Letter_Birmingham.html [consulté le 1.4.2011] (Note de la rédaction).

3. Ma traduction (S. C.-W.)

4. Ma traduction (S. C.-W.)

5. Ma traduction (S. C.-W.)

6. Ma traduction (S. C.-W.)

7. Ma traduction (S. C.-W.)

8. Ma traduction (S. C.-W.)

9. Ma traduction (S. C.-W.)

10. La recherche du bonheur 2006.

11. Dans le texte anglais.

12. Vers la fin de la lettre (98 - 99), King compose une autre phrase très longue qui met en avant une série de griefs, suivie d'une courte phrase exprimant une opinion modérée au style direct. Cette phrase n'est pas aussi longue que celle citée ci-dessus et elle n'est pas non plus sous forme de période. Mais elle illustre également bien la retenue de King.

13. Ma traduction (S. C.-W.)

\section{INDEX}

Keywords : enactment, evocation, incarnation, informal logic, King (Martin Luther), rhetoric Mots-clés : évocation, incarnation, King (Martin Luther), logique informelle, réalisation, rhétorique 